\title{
Using Electrophysiological Features in Cognitive Tasks: An Empirical Study
}

\author{
Ramla Ghali, Sébastien Ouellet, and Claude Frasson
}

\begin{abstract}
Learners' performances in intelligent tutoring systems or e-learning environments depend on various factors such as the nature of the task presented, their cognitive and affective abilities, etc. In this paper, we focus on studying in detail the variation of these different factors and more specifically the electroencephalogram (EEG metrics) and how they differ according to a category and a type of cognitive tasks. We also studied the possibility of predicting a learner's performance using feature selection and multiple regressions. Primarily, results shows that learners' scores could be predicted using in descending order the difficulty level of the task, the type of a task, the duration of a task and the EEG workload metric by building a multiple regression model that fit our data.
\end{abstract}

Index Terms-Cognitive tasks, EEG features, engagement, workload, distraction, multiple regression.

\section{INTRODUCTION}

To date, several studies have focused on intelligent tutoring systems and e-learning environments in order to offer a more reliable and adjustable learning experience according to the learners' capacities and performances. For this purpose, several approaches have been proposed in order to assess, predict and improve the learners' performance. However, in our knowledge, there is no study that takes into consideration the category or the type of a learning task. Yet, we think that learner's performances depend strongly on the category and/or the type of a proposed task.

From these approaches, we cited as an example some works in the field of artificial intelligence, human computer interaction, cognition and neuroscience [1]-[4]. These works were mainly based on the use of electrophysiological metrics and $\log$ files in order to assess more accurately certain learner's states or behaviors (emotions, workload, distraction, engagement, performance, gaming the system, off-task behavior, etc.).

In this paper, we focus only on the use of electrophysiological metrics and log files to study mainly the variation of these metrics and the learners' performance according to different types of cognitive tasks that we implemented. Electroencephalogram (EEG) was processed by applying Fast Fourier Transformation (FFT) on the signals

Manuscript received December 30, 2014; revised February 28, 2015. This work was supported by the FQRNT (Font Québécois de Recherche et de Nature et Technologies), SSHRC (Social Science and Human Research Council) through the LEADS project and NSERC (National Science and Engineering Research Council).

The authors are with the Université de Montréal, Département d'Informatique et de Recherche Opérationnelle, 2900 Chemin de la Tour, Montréal, H3C 3J7 Canada (e-mail: ghaliram@iro.umontreal.ca, sebouel@gmail.com, frasson@iro.umontreal.ca). and extracting from the latter five main power spectral densities (Delta:0-4Hz; Theta:4-8Hz; Alpha:8-12Hz; Beta: $12-40 \mathrm{~Hz}$ and Gamma: $>40 \mathrm{~Hz}$ ), and using these measures, Stevens and his colleagues has established three main metrics: mental engagement, mental workload, and distraction [5]. Mental engagement is related to the level of mental vigilance and alertness during the task (high or low states of vigilance). Mental workload can also be seen as the mental vigilance and cognitive load in a particular task. However, distraction or drowsiness reflects the feeling of being sleepy and lethargic [5].

Galvanic Skin Response (GSR) was used to assess the electrodermal activity and the skin temperature. It measures actually the individual degree of sweating, known as the excitation factor of emotions (low or strong). It is also correlated with cognitive load, stress [6] and frustration [7].

Other than the use of EEG and GSR sensors, researchers employ more complex sensors (such as eye trackers, kinect2 depth sensor, posture detection, etc.). In this paper, we use only two main sensors (EEG and GSR) and we conducted an empirical study where we collected data from our cognitive environments (composed of 7 tasks in total and 3 main categories: memory, concentration and reasoning). We also studied the variation of these outputs on the participants, and finally built a multiple regression model using the task information (difficulty level, type of a task, duration and workload EEG metric) to further predict the learners' performance in our cognitive environment.

In the following, we present previous works to our study, a description of our cognitive environment, our experiment, some exploratory and statistical results using EEG features (engagement, workload, and distraction) and GSR, and finally a linear model that uses feature selection in order to provide a moderately useful prediction of the learners' performance.

\section{PREVIOUS WORKS}

In intelligent tutoring systems and e-learning environments, the detection and assessment of some learners' states (for example: engagement, distraction, motivation, emotions, etc.) remains of a great importance in order to build more adaptive and effective human computer interaction systems. For instance, an intelligent system that is able to automatically identify if the student is engaged or not in a task and adjust its content according to this variation, would be more effective for the student and could significantly improve his performance. Moreover, an intelligent system that detects if a student is sleepy and distracted while accomplishing a task using a machine learning algorithm for a classification of this state, may be used in order to adjust its learning strategy and 
provide a learner with more suitable content and/or feedback that allows him to avoid these situations of disengagement.

In order to detect and regulate the disengagement behavior, some researchers have used log files, data mining and machine learning techniques. Among these works, we cite as an example those of Baker and his colleagues [7], [8]. In ref. [7], Baker has established a classification of a student disengagement behavior into four main categories: 1) off-task behavior, 2) gaming the system, 3) carelessness and 4) without thinking behavior. He also discussed the conditions that promoted the occurrence of each type of these behaviors and proposed some methods to detect them automatically. Whereas, in ref. [8], Baker was interested more specifically to off-task behavior that occur when a student is inactive or doing some actions which are not related to the learning content (speak to other students, stay inactive, etc.). This behavior was initially modeled by extracting certain features and using latent variable models as a machine learning technique.

Other studies have adopted a different approach by using mainly the outputs of electrophysiological sensors, such as EEG, GSR, and HR (heart rate). For example, Pope and his colleagues [1] at NASA have developed an EEG engagement index based on brainwave band power spectral densities and applied in a closed-loop system to modulate task allocation. They proved that performance in a vigilance task improved when this index was used as a criterion for switching between manual and automated piloting. Berka and her colleagues [9] explored the feasibility of monitoring EEG indices of engagement and workload acquired and measured during cognitive tests. Results showed an increase of these two indices during the encoding period of verbal and image learning and memory tests compared with the recognition period. She showed also that workload increased linearly with the level of difficulty and that the EEG measures correlated with both subjective and objective performance metrics. Mostow, Chang and Nelson [10] used EEG (single channel) to distinguish the reading of adults and children and to predict the difficulty of the text. Nourbakhch and his colleagues [11] have used GSR as an indicator of cognitive load and emotions. They have assessed GSR data from two experiments: 1) during text reading tasks and 2) during arithmetic tasks that have different levels of difficulty. From these experiments, they found a strong relationship between GSR features and mental workload. Moreover, GSR data was also used in order to detect emotions [6], [12] and stress states [7], [13]. Other studies combined the outputs of different sensors to build more reliable models. For instance, in ref. [14], a framework based on Bayesian network was built to recognize three trends of the interactions, namely: flow (a total involvement within the task), stuck (a difficulty to maintain focused attention) and off-task (a drop out from the task), as well as the emotional responses occurring subsequently. EEG and physiological devices (GSR and HR) were used and the resulting evaluations showed an accuracy rate of $82 \%$ to characterize a positive vs. a negative experience, and an accuracy ranging from $81 \%$ to $90 \%$ was achieved to assess four emotions related to the interaction namely stress, confusion, frustration and boredom.

Other than assessing learners' emotions and behaviors, some works have focused on using machine learning technique in order to predict in advance a learner's performance. For example, Beal and Galan [15] studied the use of certain mental states extracted from the electroencephalogram established by [5] as two fundamental features that predict the success or failure of the learner in the next task. These two mental states (engagement and workload) have been tested and trained on a support vector machine (SVM) to construct fairly reliable models of learner performance prediction for mathematical problems. Naser [16] used knowledge extracted from learner models to determine the academic performance of a learner in order to offer him the proper difficulty level of problems to solve. Recently, the US Army Research Laboratory (ARL) evaluated and developed concepts for two types of learner models: 1) short-term learners and 2) long-term learners. Sottilare [17] also studied the feasibility of using recorded learners' performances in the development of adaptive tutoring learning chains to design future learner models.

\section{DESCRIPTION OF OUR COGNITIVE TASKS}

In this section, we describe the cognitive environment we used to study the evolution of a learner's profile which is mainly based on three parameters: his EEG metrics, his GSR and the nature of a cognitive task presented. This environment is mainly divided in the three categories mentioned below:

\section{A. Memory}

This category contains two subcategories of the famous task of Digit Span (DS) where the learner is shown a series of numbers according to an ascending difficulty level and asked to remember and type them in the appropriate place. 1) Forward Digit Span (FDS) consists of typing the numbers in the same order that they appeared on the screen and 2) Backward Digit Span (BDS) where numbers should be entered in reverse.

\section{B. Concentration}

This category has two different tasks: 1) Feature Match and 2) Rotation. Feature Match (FM) consists in identifying whether the two images appearing on the screen are identical or not according to their forms, numbers and colors (see Fig. 1). It has also six difficulty levels (ranging from 1 to 6 ) which vary in their geometrical number and forms. However, Rotation task (RT) consists in identifying whether two images are identical or not if any rotation is allowed. It has also five difficulty level.

\section{Reasoning}

This category has three subcategories of tasks: 1) Arithmetic Addition, 2) Odd One Out and 3) Intuitive Reasoning. In the Arithmetic Addition task (AA), the learner is asked to add two numbers and the difficulty level (3 difficulty levels were implemented) increases according to the numbers of digits to add. In Odd One Out (OO), the learner has to identify the odd image from a series of nine images appearing on the screen, and has four difficulty levels. Finally, for Intuitive Reasoning (IR), it has three levels of difficulty (varying according a time constraint: unlimited, 1 minute and 
30 seconds), and 15 series in total; every level contains 5 series of exercises. Unlike other tasks, this task is based on intuitive or analogical reasoning (see Fig. 2).

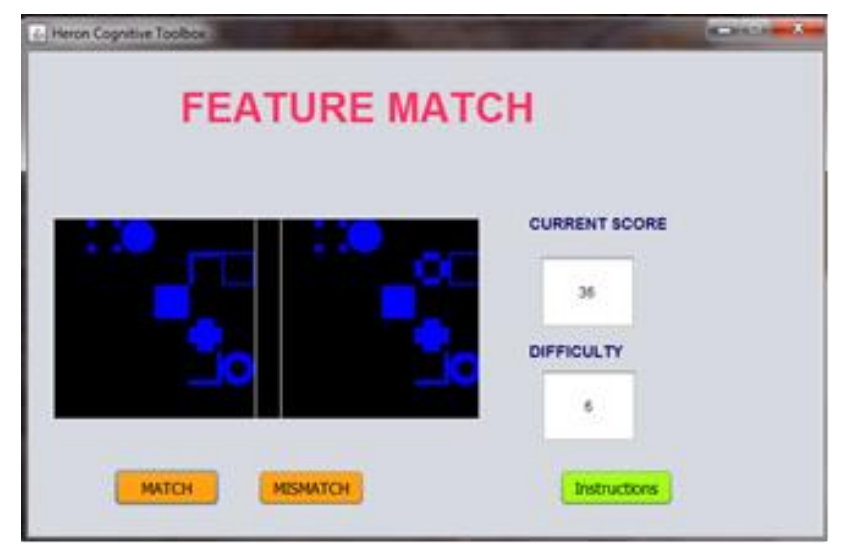

Fig. 1. Feature match task.

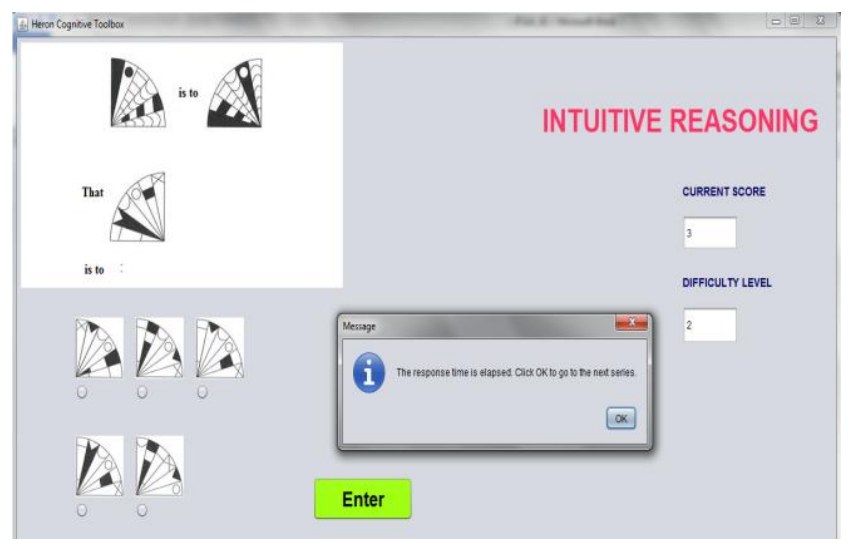

Fig. 2. An example of intuitive reasoning task.

\section{DESCRIPTION OF THE EXPERIMENT, GATHERING THE DATA AND OUR APPROACH}

\section{A. Experiment Protocol}

In order to study a learner's profile's evolution during the completion of our cognitive tasks, we conducted an experiment to gather several types of data from different subjects. In this study, all the participants (20 in total) are from the Université de Montreal. They are wearing an EEG helmet (B-Alert X10) and a bracelet (Affectiva $Q$-sensor). Participants are from different disciplines, and they are distributed as follows: 9 women and 11 men, a mean age of 28 , and a standard deviation of 4.67. The study is located in our research laboratory, where we explained the objectives of the study and asked the participants to sign a consent form (ethics agreement) before starting the experiment. The study lasted about 2 hours, during which they performed the following four steps: (1) installation of the B-Alert X10 headset on the participant (mean duration of 20-25 minutes), (2) installation of Q-sensor bracelet (about 2 minutes), (3) the participant is invited to do 3 baseline tasks defined by the manufacturer of the headset to establish a classification of mental states of engagement, workload and distraction (mean duration $\simeq 20$ minutes), and (4) the participant is finally invited to use our cognitive environment, which includes different sets of cognitive tasks as described in the previous section (mean duration $\simeq 80$ minutes). Fig. 3 illustrates the whole process.

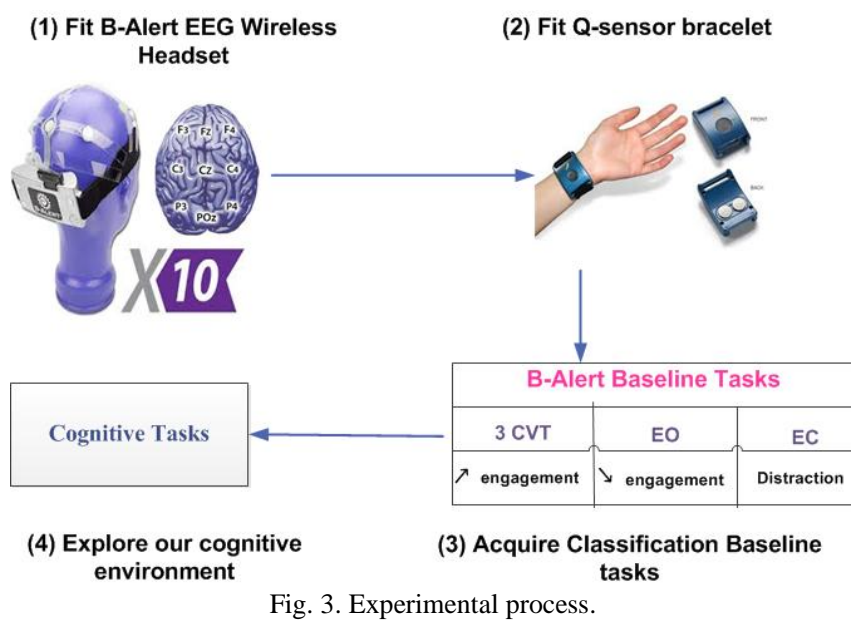

\section{B. Data Recording and Preprocessing}

The electroencephalogram (EEG) was recorded from 9 sensors (F3, Fz, F4, C3, Cz, C4, P3, Poz and P4) integrated into a cap covering the upper half of the head, with 2 reference signals attached to the mastoid bones (behind the ears). The location of each sensor was determined by the international 10-20 system. This cap was equipped with a small USB dongle which received the wireless transmissions connected to a PC computer. Each second, 256 EEG signals were transmitted and converted to power spectral densities (Alpha, Beta, Theta and Sigma) and processed by the B-Alert software which is the proprietary of Advanced Brain Monitoring Company [18]. This software allows us to obtain a real time classification of certain mental states (Sleep Onset, Distraction, Low Engagement, High Engagement and High Workload). In this study, we selected the mental states of High Engagement, Workload and Distraction. Galvanic Skin Conductance (GSR) also known as ElectroDermal Activity (EDA) was measured using Affectiva $Q$-sensor bracelet 2.0 at a sampling rate of $32 \mathrm{~Hz}$. This bracelet allows us to measure three features: skin conductance (GSR or EDA), skin temperature and an accelerometer that outputs three-dimensional displacements of the sensors. From 20 participants, only 18 data subjects were considered in the data analysis for EEG and only 16 data for GSR due to missing recordings. The EEG mental states and the GSR measures were manually synchronized with all the tasks of the cognitive environment based on the date and time extracted from log files. We calculated for each category and for each level of a task the EEG mental states values and the GSR values in order to analyze their variation depending on category and difficulty level of a task. All the data was preprocessed and labeled according to SPSS rules.

\section{Our Approach}

In this section, we show a diagram that summarizes our proposed approach (Fig. 4). We recall that we are mainly based on the study of the results from the experiment described above and the collected data from two sensors (EEG and GSR). In the first step of this approach, we gathered data from different students based on the sensors and students' interaction with our cognitive tasks (step 2). In the third step, we analyzed and studied the obtained results. Finally (step 4), we predicted learners' performances (scores) using mainly multiple regression algorithms. 


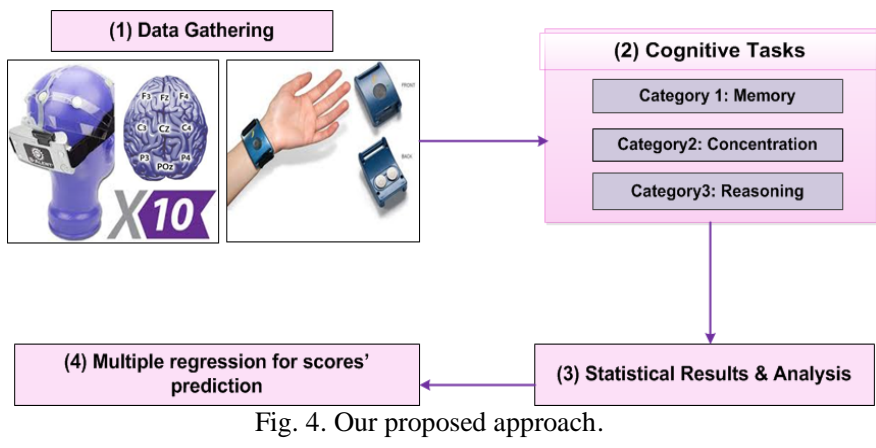

\section{EXPERIMENTAL RESULTS}

In this part, we analyze the variation of EEG and GSR metrics among all the participants. We also take into consideration the type and/or the category of a task in our study. It is mainly divided into two parts: 1) the study of EEG and GSR evolution during the cognitive tasks and 2) the possibility of predicting the learner's score using linear models such as regression.

\section{A. EEG and GSR Metrics Evolution in Learning}

In the first step, we studied the distribution of the electrophysiological metrics (EEG indices of engagement, workload and distraction) between the learners during all the cognitive tasks.

For EEG indices (distraction, engagement and workload) and for GSR metrics, we calculated the average of these metrics for each participant during the completion of our cognitive environment and the average for all the participants for each metric. We consider the latter as a threshold that we represent with a red solid line in the following figures.

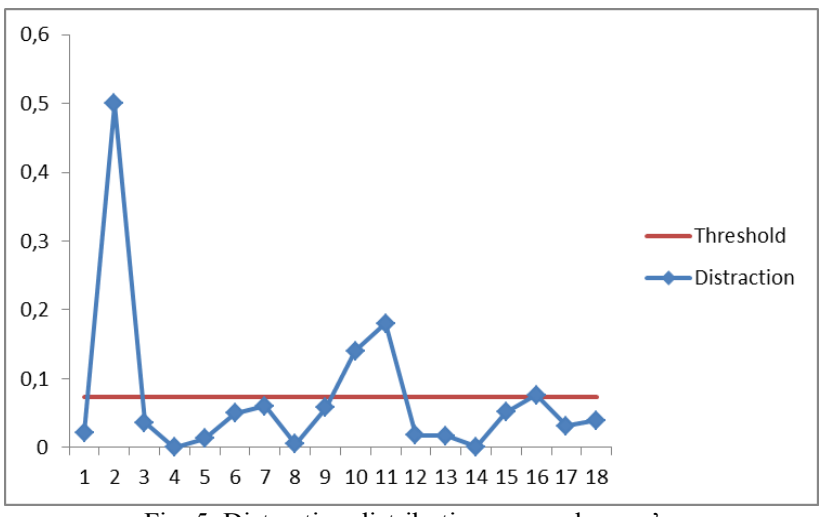

Fig. 5. Distraction distribution among learner's.

For the distraction (D) indice (Fig. 5), we notice that almost all the learners have an indice below the threshold, except the first one that has the highest value of distraction (may be due to a feeling of tiredness during the experiment). That means that almost all the learners are very awake and not sleepy during these cognitive tasks. It also shows that our tasks are interesting for the participants.

However, for the engagement (E) and workload (W) indices (see Fig. 6 and Fig. 7), we notice that theses indices vary and fluctuate according to the individual differences and situations. Therefore, some people have a high level of engagement or workload and others have low ones. So, we can say that this variation may depend on each category or type of a task but not on the overall of the cognitive environment.

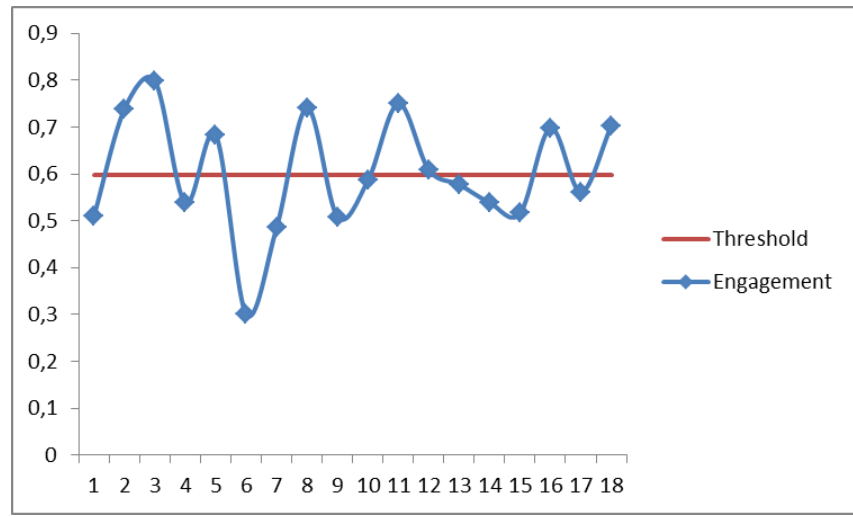

Fig. 6. Engagement distribution among learner's.

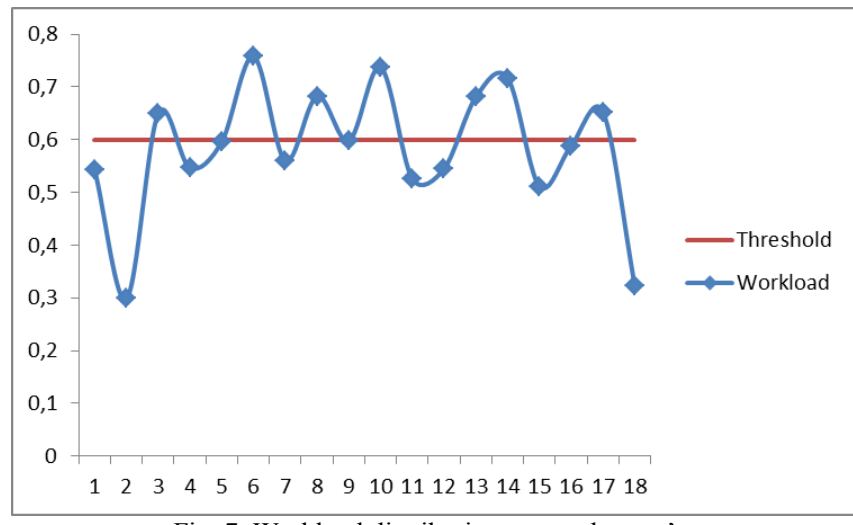

Fig. 7. Workload distribution among learner's.

To verify this hypothesis, we calculate for each task category and each task type the variation of these EEG indices (mean and standard deviation). These results are presented in the following table (Table I).

From this table, we can see clearly that the workload indice (W) has almost the highest value comparing to the other EEG metrics ( $E$ and $D)$. This means that our cognitive environment requires a minimum effort and level of mental workload to complete the tasks. Moreover, according to a comparison by the category of a task, we can see that reasoning category has the highest values of workload (0.64) and engagement (0.62) and a lower value of a standard deviation, which indicates that the data points tend to be very close to the mean. Conversely, the distraction's values are very small (near to 0 ) which results in a highest standard deviation for all the categories and types of tasks. Alike, we can notice that for the type of a task, the workload value has always the highest value except for the Arithmetic Addition (AA) and Rotation (RT) tasks. Moreover, we can see that Intuitive Reasoning (IR) task is the most difficult one because it has the highest value of workload among all types of tasks. These results lead us to accomplish two one way ANOVA tests to study if the Engagement and/or the Workload depend on a category and/or type of cognitive tasks. By realizing a one way ANOVA between the engagement EEG metric and the workload and the three categories of tasks (independent variables), we reported a very significant differences for the engagement: $\mathrm{F}(2,401)=10.89 ; p=0.00^{* *}$. Three popular post hoc tests were also taking into consideration (Least Significant Level (LSD), Tukey and Scheffe) for multiple mean comparisons and corrections. For all these three post hoc tests and for the 
engagement metric, we obtained a very significant result by comparing all the categories twice. Whereas for the workload EEG metric, it also depends on a category of a task $(\mathrm{F}(2,401)=3.9 ; p=0,02 *)$ but the results are not significant when we consider all the three post hoc tests previously mentioned. We performed likewise another ANOVA test which studied the EEG metrics and a type of a task. We find that the engagement depends on a type of a task $(\mathrm{F}(6,397)=4.95 ; p=0.00 * *)$ and the same result for the workload $(\mathrm{F}(6,397)=2.86 ; p=0.01 *)$. However, results for post hoc tests are not significant in the two cases.

TABLE I: DisTRIBUTION OF EEG METRICS ACCORDING TO CATEGORY AND TYPE OF A TASK

\begin{tabular}{|c|c|c|c|c|c|c|c|}
\hline \multirow[t]{2}{*}{ Category } & \multicolumn{3}{|c|}{ Mean (SD) } & \multirow[t]{2}{*}{ Task Type } & \multicolumn{3}{|c|}{ Mean (SD) } \\
\hline & D & $\mathbf{E}$ & W & & D & $\mathbf{E}$ & W \\
\hline \multirow[t]{2}{*}{ Memory } & \multirow[t]{2}{*}{$0.08(0.17)$} & \multirow[t]{2}{*}{$0.52(0.19)$} & \multirow[t]{2}{*}{$0.65(0.11)$} & FDS & $0.1(0.16)$ & $0.5(0.2)$ & $0.61(0.16)$ \\
\hline & & & & BDS & $0.09(0.18)$ & $0.51(0.21)$ & $0.67(0.09)$ \\
\hline \multirow[t]{2}{*}{ Concentration } & \multirow[t]{2}{*}{$0.08(0.14)$} & \multirow[t]{2}{*}{$0.56(0.18)$} & \multirow[t]{2}{*}{$0.58(0.12)$} & FM & $0.09(0.17)$ & $0.54(0.02)$ & $0.6(0.13)$ \\
\hline & & & & RT & $0.06(0.07)$ & $0.6 \quad(0.1)$ & $0.6(0.1)$ \\
\hline \multirow[t]{3}{*}{ Reasoning } & \multirow[t]{3}{*}{$0.06(0.13)$} & \multirow[t]{3}{*}{$0.62(0.19)$} & \multirow[t]{3}{*}{$0.64(0.09)$} & AA & $0.03(0.04)$ & $0.62(0.16)$ & $\mathbf{0 . 6 2}(0.12)$ \\
\hline & & & & $\mathrm{OO}$ & $0.05(0.07)$ & $0.62(0.1)$ & $0.63(0.06)$ \\
\hline & & & & IR & $0.08(0.18)$ & $0.58(0.23)$ & $\mathbf{0 . 6 5}(0.09)$ \\
\hline
\end{tabular}

After studying the behavior of EEG metrics, we analyze in a next step the GSR metric (Galvanic Skin Response) which gives an indication of emotions valence or a level of stress [6], [7]. The figure below illustrates the variation of this metric accounting the average values for each participant and a threshold computed by considering the average of all the data as we mentioned in the last section.

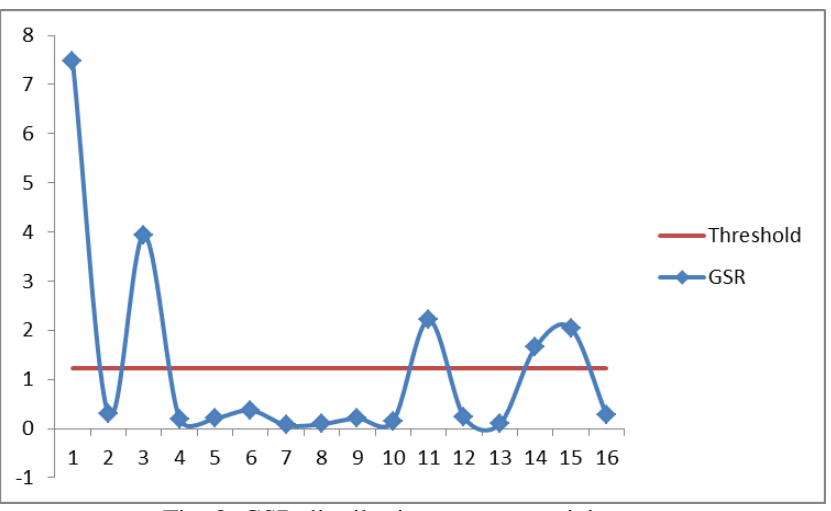

Fig. 8. GSR distribution among participants.

From this figure, we realize that almost the values are under the threshold which is also very low comparing to other studies (1.22). We can see that we have only five values that we can take into consideration for more statistical analysis, which is a small number of data and we can't apply a technique to change these values according to the data distribution (such as Expectation Maximization technique or Multiple Imputation) due to this small sample of data. This result could be explained by a technical problem occurring during recording the learners' GSR or the individual difference reactions to this metric [11], [19]. For this reason, we don't focus too much to study the relationship between this metric and the different tasks of our cognitive environment. We think rather that we don't have a reliable dataset for this purpose.

\section{B. Multiple Regression for Predicting Learners' Scores}

After studying the electrophysiological metrics and their influence depending on a category and a type of task, we focus next to find a reliable function that connects the electrophysiological variables (EEG indices of workload (W), engagement (E) and distraction (D)) and task information (task duration, task level: ranging from 1 to 6 and task type) in order to predict learners' score.

For this purpose, we performed cross tables that studied the Pearson correlation between the score (the dependent variable) and the others variables (engagement, workload, the distraction, the type, the duration and the level of the task). Results from this tables show a very low correlation between the score and the distraction $(R=0.036 ; p=0.233>5 \%)$. That means that there isn't any correlation between these two variables and the distraction logically could be eliminated if we want to predict the score using fewer features. We obtained significant results for the EEG metrics and the task information: type of a task (TT), level of difficulty (DL) and duration of completing the task (D). Table II reports in details the values obtained for the different metrics using mainly Pearson's Correlation.

TABLE II: RESULTS OF PEARSON CORRELATIONS

\begin{tabular}{|c|c|c|}
\hline Pearson Correlation & \multicolumn{2}{|c|}{ Score } \\
\hline Results & $R$ & $p$ \\
\hline Distraction (D) & 0.036 & 0.233 \\
\hline Engagement (E) & -0.118 & $0.009 * *$ \\
\hline Workload (W) & -0.122 & $0.007 * *$ \\
\hline Task Type (TT) & -0.13 & $0.004 * *$ \\
\hline Difficulty Level (DL) & -0.382 & $0.000 * *$ \\
\hline Duration (D) & -0.125 & $0.006^{* *}$ \\
\hline
\end{tabular}

where $* *$ presents a significantly level at $1 \%$, and $*$ at $5 \%$.

This table gives us a brief idea which variables are independent from the score and could be used further to fit a linear regression curve according to these values.

We performed next a linear regression using multiple variables and using a stepwise method to establish the best model that fit our data. Using EEG indices and task information, we obtained the best model with a coefficient of correlation $R$ equal to 0.501 (medium correlation) and a Dubin-Watson value equal to 1.34 which is in the interval of $[0,4]$ and near to 2 . Our model is constructed in descending order by the difficulty level of a task, the task type, the duration of the task and the workload EEG metric (see Table III).

From this table, we can deduce the equation of a predicted score: 
TABLE III: MULTIPLE REGRESSION MODEL TO PREDICT LEARNERS' SCORES

\begin{tabular}{|l|l|l|l|l|}
\hline \multirow{2}{*}{$\begin{array}{c}\text { Model } \\
\text { Components }\end{array}$} & \multicolumn{4}{|c|}{ Coefficients } \\
\cline { 2 - 5 } Constant & 125.89 & & 17.02 & $0.000^{* * *}$ \\
\hline $\begin{array}{l}\text { Difficulty } \\
\text { Level (DL) }\end{array}$ & -8.667 & -0.484 & -10.4 & $0.000^{* *}$ \\
\hline $\begin{array}{l}\text { Task Type } \\
\text { (TT) }\end{array}$ & -4.304 & -0.293 & -6.3 & $0.000^{* *}$ \\
\hline Duration (D) & -0.002 & $-0,122$ & -2.81 & $0.005^{* *}$ \\
\hline Workload (W) & -23.268 & $-0,099$ & -2.27 & $0.024^{*}$ \\
\hline
\end{tabular}

score $=125.89-8.667 D L-4.304 T T-0.002 D-32.268 \mathrm{~W}$

This equation (1) indicates that all our variables have a negative sign indicating an inverse relationship between those independent variables and the dependent one (score).

Finally, in order to study the role of task information, we conducted another linear regression using only the EEG metrics, more precisely the engagement and workload. By eliminating the task information, we noticed that correlation indices $\mathrm{R}$ decreased to more than three times $(\mathrm{R}=0.162)$. We noticed also that the $\mathrm{p}$-value increases when constructing the model. However, using only this information, the score could be predicted as follows:

$$
\text { score }=95.4-26.32 \mathrm{~W}-15.75 E
$$

This second equation (2) confirms too that the score has a negative relationship with EEG metrics.

\section{CONCLUSION}

In this paper, we conducted an empirical study of how to include EEG features depending on a category or a type of cognitive task. For GSR feature, we don't have unfortunately correct values from all the participants and the reliable values are not enough to establish a conclusion of how it varies according to a type of cognitive task. We also noticed a very significant result between the engagement and the category of a task. This result is confirmed by applying three post hoc tests (LSD, Tukey and Scheffe). Moreover, we noticed a strong negative correlation between engagement, workload and task information (difficulty level, task type and duration). The latter leads us to build a linear regression model that fits our dataset, and we successfully obtained an inverse relationship with the score (the variable to predict). As a conclusion, we can moderately predict the score using a multiple regression model that is constructed in descending order of relevance by the difficulty level, the task type, the duration of a task and the EEG workload feature.

Nevertheless, our work has some limitations. For instance, we can improve the prediction of learners' scores by using more complex machine learning techniques (nonlinear models, decision trees, Adaboost, etc.). We also can integrate the best model in real time estimation and regulation of learning content in order to improve the low scores. We can also develop pedagogical strategies in order to deal with learner's performances and the type of a cognitive task.

\section{REFERENCES}

[1] A. T. Pope, E. H. Bogart, and D. S. Bartolome, "Biocybernetic system evaluates indices of operator engagement in automated task," IEEE Trans. Electron Devices, vol. ED-11, pp. 34-39, Jan. 1995.
[2] G. F. Wilson, "An analysis of mental workload in pilots during flight using multiple electrophysiological measures," International Journal Aviation Psychology, vol. 12, pp. 3-18, 2004.

[3] S. D’Mello, R. Taylor, K. Tapp, B. King, and A. Graesser, "Posture as a predictor of learners' affective engagement: Boredom and flow," presented at Annual AERA Meeting, 2007.

[4] R. S. Baker and L. M. Rossi, "Assessing the disengaged behaviors of learners," Design Recommendations for Intelligent Tutoring Systems, vol. 1, ch. 14, 2013.

[5] R. Stevens, T. Galloway, and C. Berka, "EEG-related changes in cognitive workload, engagement and distraction as students acquire problem solving skills," in User Modeling, C. Conati, K. McCoy, and G. Paliouras, ed., Heidelberg: Springer, Berlin, pp. 187-196, 2007.

[6] Y. Mohammad and T. Nishida. "Using physiological signals to detect natural interactive behavior," Applied Intelligence, vol. 33, no. 1, pp. 79-92, 2010

[7] B. Winslow, "Affective learning companions: strategies for empathetic agents with real-time multimodal affective sensing to foster meta-cognitive and meta-affective approaches to learning, motivation, and perseverance," Ph.D. dissertation, Dept. Physics. Eng., Massachusetts Institute of Technology, MIT Media lab., Cambridge, USA, 2006.

[8] R. S. J. D. Baker, "Modeling and understanding students' off-task behavior in intelligent tutoring systems," in Proc. the SIGCHI Conference on Human Factors in Computing Systems, San Jose, California, USA, 2007, pp. 1059-1068.

[9] C. Berka, D. J. Levendowski, M. M. Cvetinovic et al., "Real-time analysis of eeg indexes of alertness, cognition, and memory acquired with a wireless EEG headset," International Journal of Human-Computer Interaction , vol. 17, pp. 151-170, 2004.

[10] J. Mostow, K. Chang, and J. Nelson, "Towards exploiting EEG input in a reading tutor," in Proc. AIED Conference, Springer Verlag, 2011, pp. 230-237.

[11] N. Nourbakhsh, Y. Wang, F. Chen, and R. Clavo, "Using galvanic skin response for cognitive load measurement in arithmetic and reading tasks," in Proc. the 24th Australian Computer-Human Interaction Conference, Melbourne, Australia, 2012, pp. 420-423.

[12] A. Nakasone, H. Prendinger, and M. Ishizuka, "Emotion recognition from electromyography and skin conductance," presented at the Fifth International Workshop on Biosignal Interpretation, 2005.

[13] C. Setz, B. Arnrich, J. Schumm, R. L. Marca, G. Tröster, and U. Ehlert, "Discriminating stress from cognitive load using a wearable EDA device," IEEE Trans Inf. Technol. Biomed., vol. 14, no. 2, pp. 410-7, Nov. 2010.

[14] I. Jraidi, M. Chaouachi, and C. Frasson, "A dynamic multimodal approach for assessing learner's interaction experience," presented at 15th ACM International Conference on Multimodal Interaction (ICMI2013), Sydney, Australia, December 9-13, 2013.

[15] C. Beal and C. Galan, "EEG estimates of engagement and cognitive workload predict math problem solving outcomes," presented at User Modeling And Personalization Conference, Montreal, Canada, 2012.

[16] S. A. Naser, "Predicting learners performance using artificial neural networks in linear programming intelligent tutoring systems," International Journal of Artificial Intelligence and Applications, vol. 3, no. 2, March 2012.

[17] R. A. Sottilare, "Using learner data to influence performance during adaptive tutoring experiences," Foundations of Augmented Cognition, Advancing Human Performance and Descision-Making through Adaptive Systems. Lectures notes in Computer Science, vol. 85, no. 34, pp. 265-275, 2014.

[18] Advanced brain monitoring. [Online]. Available: http://advancedbrainmonitoring.com/xseries/x10/

[19] E. Haapalainen, S. Kim, J. F. Forlizzi, and A. K. Dey, "Psycho-physiological measures for assessing cognitive load," in Proc the $12^{\text {th }}$ ACM International Conference on Ubiquitous Computing, Copenhagen, Denmark, 2010, pp. 301-310.

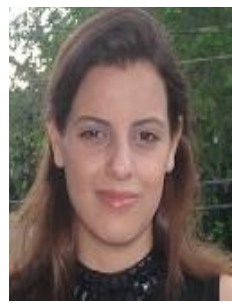

Ramla Ghali was born in Ariana (Tunisia). Ghali is currently a Ph.D student under the supervision of Prof. Claude Frasson at University of Montreal (Canada). In 2010, Ghali had her master's degree in computer science from Udem and a bachelor degree in the same field 2006 from the Faculty of Computer Science in Monastir (Tunisia). Ghali was focused on studying the variation of different electrophysiological sensors in cognitive environment depending on a type of a task during interacting with learning environments. She is also interested in intelligent learning environments which involves a cognitive load and used 
several machine learning techniques in order to establish a real time classification and/or regression of learners' performance to provide the latter with more adaptability and feedback.

She is also a member of LEADS project (Learning Environment Across Disciplines) on collaboration with McGill Univeristy and a research assistant at Héron and GRITI laboratory. She was also a lecturer of Matlab Programming course at École Polytechnique de Montréal (Fall 2012) and a laboratory assistant for this course for a great period of time. She taught also database course as an assistant laboratory (Fall 2013 and winter 2014).

Ramla Ghali had received an excellence award from FQRNT (Font Québécois de Recherche et de Nature et Technologies) at 2013 for her Ph. D. studies and an excellence award from DIRO (Département d'Informatique et de Recherche Opérationnelle, Université de Montréal) in April 2014.

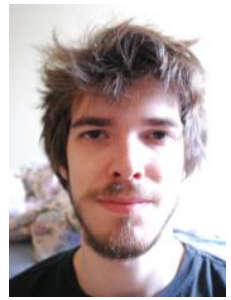

Sébastien Ouellet is a master's student in compute science at the Université de Montreal. He studied cognitive science at Carleton University and was a member of the Science of Imagination Laboratory, taking part in artificial intelligence projects supported by psychological data with a focus on models of spatial and visual concepts. He is now a member of the Jeux Vidéo Intelligents (JVI) laboratory, where he is involved with the development of educational games that merge machine learning and physiological sensing. Interdisciplinary research is a cornerstone of his interests, alongside the use of AI in games, whether it is for cooperative or competitive purposes.

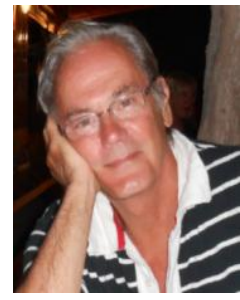

Claude Frasson is a professor in computer science at University of Montreal since 1983, the head of the HERON laboratory and GRITI inter-university Research Group involving seven universities in Quebec. His research interests are at the convergence of artificial intelligence, education, human computer interaction. He founded, in 1988, the ITS international conference in Intelligent Tutoring Systems, which holds every two years. He coordinated the SAFARI project, a multidisciplinary research effort involving an industrial approach for building, distributing and controlling intelligent courses on the Web and is at the origin of a patent on a distance learning architecture based on networked cognitive agents. He received an ACM award for excellence as the organization chair of 2001 Autonomous Agent Conference. Since 2004, he aims to understand how the human brain functions with emotions, considering that they play an important role in knowledge acquisition, and subconscious learning, using EEG systems. 